\title{
COUNTABLE METACOMPACTNESS IN $\Psi$-SPACES
}

\author{
PAUL J. SZEPTYCKI
}

(Communicated by Andreas R. Blass)

\begin{abstract}
We prove under a variety of assumptions including $c=\aleph_{2}$ that, for every maximal almost disjoint family $\mathscr{A}$ of countable subsets of $\omega_{1}, \Psi(\mathscr{A})$ is not countably metacompact. In addition, a first countable, countably metacompact, regular space with a closed discrete set which is not a $G_{\delta}$ is constructed from the mutually consistent assumptions that $\mathfrak{b}=\omega_{1}$ and there can exist a Q-set.
\end{abstract}

\section{INTRODUCTION}

Recall that a space is perfect if each closed subset is a $G_{\delta}$. The easy but important result that all perfect spaces are countably metacompact raises the natural question: how perfect are countably metacompact spaces? In [Bu2] Burke proved that under PMEA closed discrete sets are $G_{\delta}$ 's in first countable countably metacompact $T_{1}$ spaces.

Given a maximal almost disjoint (mad) family $\mathscr{A} \subseteq\left[\omega_{1}\right]^{\omega}$ we define the space $\Psi(\mathscr{A})$ as: $\omega_{1} \cup \mathscr{A}$ is the underlying set. Every point in $\omega_{1}$ is isolated while a typical neighborhood of an $a \in \mathscr{A}$ looks like $\{a\} \cup a \backslash y$ where $y$ is a finite subset of $a$. Then $\Psi(\mathscr{A})$ is a regular, first countable space and $\mathscr{A}$ is a closed discrete set which is not a $G_{\delta}$. So if there exists a mad $\mathscr{A}$ such that $\Psi(\mathscr{A})$ is countably metacompact, there would be a nice counterexample to the PMEA result. In [Bu1] Burke raised this question and answered it negatively under the assumption $a=c$. In this note we again answer the question in the negative under a number of different assumptions, including $\mathfrak{c}=\aleph_{2}$.

There are only two consistent counterexamples to Burke's PMEA theorem in the literature. In [Sh] Shelah forced a normal countably metacompact ladder system space with a closed discrete set which is not a $G_{\delta}$, while in [BBu] Balogh and Burke constructed a regular counterexample in a ccc forcing extension. Assuming $\mathfrak{b}=\omega_{1}$ and there can exist a Q-set, we construct a regular first countable countably metacompact space with a closed discrete set which is not a $G_{\delta}$. This is the only known regular counterexample to the PMEA result which is not a forcing construction.

Received by the editors September 30, 1991 and, in revised form, July 10, 1992; presented to the Summer Conference on General Topology and Applications, June 26-29, 1991, University of Wisconsin, Madison.

1991 Mathematics Subject Classification. Primary 03E05, 54D18, 54G20.

Key words and phrases. Countably metacompact, perfect, mad, unbounded, Q-set. 
Our terminology and notation are fairly standard. $\left[\omega_{1}\right]^{\omega}=$ the collection of countably infinite subsets of $\omega_{1} . \mathscr{A}$ indicates an infinite mad family on $\left[\omega_{1}\right]^{\omega}$. $\mathfrak{a}$ is defined as the minimal cardinality of an infinite mad family on $\omega$. For $f, g \in{ }^{\omega} \omega, f \leq^{*} g$ means $g(n)>f(n)$ for at most finitely many $n$. b is the minimal cardinality of an unbounded family in $\left({ }^{\omega} \omega, \leq^{*}\right)$, and $\mathfrak{d}$ is the minimal cardinality of a dominating family in $\left({ }^{\omega} \omega, \leq^{*}\right) . X \subseteq^{*} Y$ means that $X \backslash Y$ is finite. Given $Y \subseteq \omega_{1}, \mathscr{A} \mid Y=\left\{a \cap Y: a \in \mathscr{A}\right.$ s.t. $\left.|a \cap Y|=\aleph_{0}\right\}$ and $\Psi(\mathscr{A} \uparrow Y)$ is the subspace of $\Psi(\mathscr{A})$ determined by $Y \cup(\mathscr{A} \uparrow Y)$. For more on $\mathfrak{b}, \mathfrak{d}, \mathfrak{a}$, and $\Psi(\mathscr{A})$ see $[\mathrm{vD}]$.

\section{Preliminaries}

We will use the following formulation of countable metacompactness.

Definition 2.1. A space $X$ is countably metacompact iff for every decreasing sequence $\left\{D_{n}: n<\omega\right\}$ of closed subsets of $X$ such that $\bigcap_{n<\omega} D_{n}=\varnothing$ there exist open $U_{n} \supseteq D_{n}$ such that $\bigcap_{n<\omega} U_{n}=\varnothing$.

The following lemma follows directly from the definitions.

Lemma 2.2. Given a mad $\mathscr{A} \subseteq\left[\omega_{1}\right]^{\omega}, \Psi(\mathscr{A})$ is countably metacompact iff for every partition $\left\{\mathscr{A}_{n}: n \in \omega\right\}$ of $\mathscr{A}$ there are $X_{n} \subseteq \omega_{1}$ such that $\forall n \forall m \geq$ $n \forall a \in \mathscr{A}_{m} \quad\left(a \subseteq^{*} X_{n}\right.$ and $\left.\bigcap_{n<\omega} X_{n}=\varnothing\right)$.

Theorem 2.3. Suppose c is regular. Given a mad $\mathscr{A} \subseteq\left[\omega_{1}\right]^{\omega}$, if $\exists Z \in\left[\omega_{1}\right]^{\omega_{1}}$ such that $|\mathscr{A}| Y \mid=\mathfrak{c}$ for each $Y \in[Z]^{\omega_{1}}$, then $\Psi(\mathscr{A})$ is not countably metacompact.

Proof. Enumerate $\left\{x \in\left[\omega_{1}\right]^{\omega}:|\mathscr{A}| x \mid=\mathfrak{c}\right\}$ as $\left\{x_{\alpha}: \alpha \leq \mathfrak{c}\right\}$. Notice that for each $X \in\left[\omega_{1}\right]^{\omega_{1}}$ if $|\mathscr{A}| X \mid=\mathfrak{c}$ then there is an $x \in[X]^{\omega}$ s.t. $|\mathscr{A}| x \mid=\mathfrak{c}$. For each $n \in \omega$ we construct $\mathscr{A}_{\alpha}^{n} \subseteq \mathscr{A}$ inductively on $\alpha \in \mathfrak{c}$ as follows. Fix $a_{n} \in \mathscr{A}$ such that $\forall n \neq m \quad a_{n} \neq a_{m}$, and $\forall n \quad\left|a_{n} \cap x_{o}\right|=\aleph_{0}$. Let $\mathscr{A}_{0}^{n}=\left\{a_{n}\right\}$.

Having defined $\mathscr{A}_{\beta}^{n}$ for all $\beta \leq \alpha$ s.t.

(i) $\left|\mathscr{A}_{\beta}^{n}\right|=|\beta|$,

(ii) $\forall n \neq m \mathscr{A}_{\beta}^{n} \cap \mathscr{A}_{\beta}^{m}=\varnothing$,

(iii) $\forall \beta \in \alpha \exists a \in \mathscr{A}_{\beta}^{n}\left|a \cap x_{\beta}\right|=\aleph_{0}$.

Let $\mathscr{A}^{\prime}=\bigcup_{\beta \in \alpha}\left\{\mathscr{A}_{\beta}^{n}: n \in \omega, \beta \in \alpha\right\}$. For each $n \in \omega$ choose $a_{\alpha}^{n} \in \mathscr{A}-\mathscr{A}^{\prime}$ distinct such that $\left|a_{\alpha}^{n} \cap x_{\alpha}\right|=\aleph_{0}$. Let $\mathscr{A}_{\alpha}^{n}=\bigcup_{\beta \in \alpha} \mathscr{A}_{\beta}^{n} \cup\left\{a_{\alpha}^{n}\right\}$. Finally let $\mathscr{A}_{n}=\bigcup_{\alpha \in \mathfrak{c}} \mathscr{A}_{\alpha}{ }^{n}$. Notice that if $x \in\left[\omega_{1}\right]^{\omega}$ is such that $|\mathscr{A}| x \mid=\mathfrak{c}$ then for each $n$ there is an $a \in \mathscr{A}_{n}$ such that $|a \cap x|=\aleph_{0}$. Fix $n \in \omega$. If $X$ is such that $\forall m \geq n \forall a \in \mathscr{A}_{m} a \subseteq^{*} X$, then $|Z-X|$ is countable. Hence, the $\mathscr{A}_{n}$ witness that $\Psi(\mathscr{A})$ is not countably metacompact.

The following is a corollary to the proof of Theorem 2.3.

Corollary 2.4 (Burke). $(\mathfrak{a}=\mathfrak{c}) \rightarrow \Psi(\mathscr{A})$ is not countably metacompact for every $\operatorname{mad} \mathscr{A} \subseteq\left[\omega_{1}\right]^{\omega}$.

\section{UNBOUNDED FAMILIES AND PARTITIONS OF MAD FAMILIES}

By Lemma 2.2 , to prove that $\Psi(\mathscr{A})$ is not countably metacompact for some mad $\mathscr{A}$ we must exhibit a nasty (i.e., witnessing not countable metacompactness) partition of $\mathscr{A}$ into countably many pieces. We do this by indexing $\mathscr{A}$ 
with a family $\mathscr{F} \subseteq{ }^{\omega} \omega$ and proving that if $\mathscr{F}$ has certain nice properties then we can build a nasty partition for $\mathscr{A}$. A similar technique was used by Simon in $[\mathrm{S}]$ to build a Frechet space whose square is not Frechet.

Definition 3.1. Given $\mathscr{F} \subseteq{ }^{\omega} \omega$, we say $\mathscr{F}$ is fully unbounded if $\forall S \in[\mathscr{F}]^{|\mathscr{F}|}$ $S$ is unbounded under $\leq^{*}$.

Recall that if $f, g \in{ }^{\omega} \omega$, then $f \leq^{*} g \Leftrightarrow\{n: g(n)>f(n)\}$ is finite. Clearly no family of size $\kappa$ where $\kappa<\mathfrak{b}$ or $\kappa$ is regular and $>\mathfrak{d}$ can be fully unbounded. However, if $\mathfrak{b} \leq \kappa \leq \mathfrak{d}$ then we have positive, consistent, and independent results.

Theorem 3.2. (i) There are fully unbounded families of size $\mathfrak{d}$ and $\mathfrak{b}$.

(ii) Let $\kappa<\delta<\lambda$ be regular uncountable cardinals. Then $\operatorname{Con}(\mathrm{ZFC}) \rightarrow$ $\operatorname{Con}(\mathrm{ZFC}+(\mathfrak{b}=\kappa)+(\mathfrak{d}=\lambda)$ and there is no fully unbounded family of size $\delta)$.

(iii) Let $\kappa$ be a regular uncountable cardinal. Then Con(ZFC) $\rightarrow$ $\operatorname{Con}\left(\mathrm{ZFC}+\left(\mathfrak{b}=\aleph_{1}\right)+(\mathfrak{d}=\mathfrak{c}=\kappa)\right.$ and there is a fully unbounded family of size $\delta$ for each uncountable $\delta<\mathfrak{c}$ ).

Proof. (i) Fix a well-ordered unbounded family of type $\mathfrak{b}$ and a dominating family $\left\{f_{\alpha}: \alpha<\mathfrak{d}\right\}$ such that $\alpha<\beta \rightarrow f_{\beta} \mathbb{Z}^{*} f_{\alpha}$. Then both families are fully unbounded.

(ii) We start with a model $M$ of $\mathrm{CH}$ and iterate the dominating real poset along the well-founded poset $(\kappa \times \lambda, \preceq)$, where $(\alpha, \beta) \preceq(\gamma, \eta)$ iff $\alpha \leq \gamma$ and $\beta \leq \eta$. This is Hechler's model [H] for cofinally embedding $\kappa \times \lambda$ into $\left({ }^{\omega} \omega,<^{*}\right)$. If $f_{(\alpha, \beta)}$ is the $(\alpha, \beta)$ th function added, then $(\alpha, \beta) \preceq(\gamma, \eta)$ and $(\alpha, \beta) \neq(\gamma, \eta)$ implies that $f_{(\alpha, \beta)}<{ }^{*} f_{(\gamma, \eta)}$. Let $\mathscr{F} \subseteq{ }^{\omega} \omega$ be of size $\delta$. For each $f \in \mathscr{F}$ there is $\left(\alpha_{f}, \beta_{f}\right)$ s.t. $f \in M\left[G_{\left(\alpha_{f}, \beta_{f}\right)}\right]$. Fix $\alpha$ such that $\mathscr{F}^{0}=\left\{f: \alpha_{f}=\alpha\right\}$ has size $\delta$. Then there is a $\beta$ above $\left\{\beta_{f}: f \in \mathscr{F} 0\right\}$. Therefore, $\mathscr{F}^{0} \subseteq M\left[G_{(\alpha, \beta)}\right]$, which implies that $\mathscr{F}^{0}<^{*} f_{(\alpha, \beta)}$. Therefore, $\mathscr{F}$ is not fully unbounded.

(iii) Start with a model of $\mathrm{MA}+\mathrm{c}=\kappa$ and add $\kappa$ Cohen reals. It is straightforward to prove that $\mathfrak{b}=\aleph_{1}, \mathfrak{d}=\mathfrak{c}=\kappa$, and for any $\delta \leq \kappa$ uncountable the family consisting of the first $\delta$ Cohen reals is fully unbounded.

Theorem 3.3. Let $\mathscr{A} \subseteq\left[\omega_{1}\right]^{\omega}$ be mad such that

$$
\forall x \in\left[\omega_{1}\right]^{\omega} \quad|\mathscr{A}| x\left|\geq \aleph_{0} \rightarrow\right| \mathscr{A}|x|=|\mathscr{A}|=\kappa .
$$

Assume further that there exists an $\mathscr{F} \subseteq{ }^{\omega} \omega$ such that $|\mathscr{F}|=\kappa$ and $\mathscr{F}$ is fully unbounded; then $\Psi(\mathscr{A})$ is not countably metacompact.

Proof. Fix $\mathscr{F}$ as in the hypothesis of the theorem and index $\mathscr{A}$ as $\left\{a_{f}: f \in\right.$ $\mathscr{F}\}$. For each $n, m \in \omega$, let $\mathscr{A}_{m}^{n}=\left\{a_{f}: f(n)=m\right\}$; then, for each $n$, $\mathscr{A}=\bigcup_{m<\omega} \mathscr{A}_{m}^{n}$ is a partition of $\mathscr{A}$. Assume $\Psi(\mathscr{A})$ is countably metacompact; then for each $n$ there is $\left\langle U_{m}^{n}\right\rangle_{m<\omega} \subseteq\left[\omega_{1}\right]^{\omega_{1}}$ such that $\bigcap_{m<\omega} U_{m}^{n}=\varnothing$ and, $\forall m \forall k>m \forall a \in \mathscr{A}_{k}^{n}, a \subseteq^{*} U_{m}^{n}$.

For each $n$, choose $h(n)$ inductively such that $\left|\bigcap_{k \leq n} \omega_{1} \backslash U_{h(k)}^{k}\right|=\aleph_{1}$. Clearly this can be done since $\forall n \bigcup_{m<\omega} \omega_{1} \backslash U_{m}^{n}=\omega_{1}$. Construct $x \in\left[\omega_{1}\right]^{\omega}$ such that $\forall k x \subseteq^{*} \omega_{1} \backslash U_{h(k)}^{k}$ and such that $|\mathscr{A}| x|=| \mathscr{A} \mid$ as follows:

Let $y_{0}$ be a pseudo-intersection of $\left\{\omega_{1} \backslash U_{h(j)}^{j}\right\}_{j<\omega}$ and pick $a_{0} \in \mathscr{A}$, such that $\left|a_{0} \cap y_{0}\right|=\aleph_{0}$. Having constructed $\left\{y_{k}: k<n\right\}$ and $\left\{a_{k}: k<n\right\}$, such 
that

(i) $y_{k} \subseteq U_{h(k)}^{k}$,

(ii) $y_{k}$ is a pseudo-intersection of $\left\{\omega_{1} \backslash U_{h(j)}^{j}\right\}_{j<\omega}$,

(iii) $\left|a_{k} \cap y_{k}\right|=\aleph_{0}$, and

(iv) $i \neq k$ implies $a_{i} \neq a_{k}$,

let $y_{n}$ be a pseudo-intersection of $\left\{\omega_{1} \backslash U_{h(i)}^{i}\right\}_{i<\omega}$ with $y_{n} \subseteq U_{h(n)}^{n}$ and such that every ordinal in $y_{n}$ is above every ordinal in $\bigcup_{k<n} \alpha_{k}$. Pick $a_{n} \in \mathscr{A}$ such that $\left|a_{n} \cap y_{n}\right|=\aleph_{0}$. Let $x=\bigcup_{n<\omega} y_{n}$. Then $\forall k<\omega\left|a_{k} \cap x\right|=\aleph_{0}$. Therefore, $|\mathscr{A}| x \mid \geq \aleph_{0}$; hence, by assumption $|\mathscr{A}| x \mid=\kappa$.

Since $\mathscr{F}$ is fully unbounded, fix $f \in \mathscr{F}$ and $n \in \omega$ such that $\left|a_{f} \cap x\right|=\aleph_{0}$ and $h(n)<f(n)$. Therefore, $a_{f} \subseteq^{*} U_{h(n)}^{n}$, contradicting that $a_{f} \cap x$ is infinite and $x \cap U_{h(n)}^{n}$ is finite.

Corollary 3.4. If $\mathfrak{c}=\aleph_{2}$ or if $\mathfrak{b}^{+}=\mathfrak{c}$, then, for each $\mathscr{A} \subseteq\left[\omega_{1}\right]^{\omega} \operatorname{mad}, \Psi(\mathscr{A})$ is not countably metacompact.

Proof. Let $\mathscr{A}$ be mad. Then, since $\mathfrak{b} \leq \mathfrak{a}$, either $\forall Y \in\left[\omega_{1}\right]^{\omega_{1}}|\mathscr{A}| Y \mid=\mathfrak{c}$, or $\exists X \in\left[\omega_{1}\right]^{\omega_{1}}$ such that $\forall Y \in[X]^{\omega}|\mathscr{A}| Y \mid=\mathfrak{b}$. In the first case Theorem 2.3 implies $\Psi(\mathscr{A})$ is not countably metacompact, while the second case follows from Theorem 3.3 and the existence of a fully unbounded family of size $\mathfrak{b}$.

Theorem 3.3 suggests the following question: Does the existence of a mad family of size $\kappa$ imply the existence of a fully unbounded family of size $\kappa$ ? Since there are no fully unbounded families of regular size $>\mathfrak{d}$, the question is only interesting for mad families of singular cardinality or of size $\leq \mathfrak{d}$.

\section{A CONSISTENT COUNTEREXAMPLE}

We present in this section the construction of a regular, first countable, countably metacompact space $X$ with a closed discrete subset which is not a $G_{\delta}$. The space is constructed under the consistent assumption that $\mathfrak{b}=\omega_{1}$ and there exists a Q-set.

Definition 4.1. An uncountable subset of the reals is called a Q-set if every subset is a relative $G_{\delta}$.

The following lemma was proved by Todorcevic (see [T, Lemma 2.5]). The set function $H$ was used there to construct, among other things, a compact $S$-space from the assumption $\mathfrak{b}=\aleph_{1}$.

Lemma 4.2 (Todorcevic). Assume $\mathfrak{b}=\omega_{1}$. Fix $Z \subseteq{ }^{\omega} \omega$. There is a set function $H: Z \rightarrow[Z]^{\leq \omega}$ such that, for each $z \in Z, H(z)$ is either finite or a sequence converging to $z$ with the property that, if $Y$ and $D$ are subsets of $Z$ such that $Y \subseteq \bar{D}$ and $Y$ is uncountable, then $\{y \in Y: H(y) \cap D$ is finite $\}$ is countable.

Fix $Z \subseteq{ }^{\omega} \omega$ a Q-set of size $\aleph_{1}$. We define a topology on $X=Z \times 2$ using $H$ by letting $Z \times\{0\}$ be isolated in $X$, and a typical neighborhood of $(z, 1)$ looks like $\{(z, 1)\} \cup[H(z) \backslash F] \times\{0\}$ for some finite set $F$. Clearly $X$ with this topology is first countable and regular. The fact that $Z$ is a $Q$-set implies that the space is countably metacompact while the set function $H$ assures that $Z \times\{1\}$ is not a $G_{\delta}$ in $X$.

Claim 4.3. $X$ is countably metacompact. 
Proof. Suppose $Z_{0} \supseteq Z_{1} \supseteq \cdots$ is a sequence of subsets of $Z$ such that $\bigcap_{i<\omega} Z_{i}=\varnothing$. So $\left\{Z_{i} \times\{1\}: i<\omega\right\}$ is a typical decreasing sequence of closed sets in $X$. We use the fact that each $Z_{i}$ is a $G_{\delta}$ in $Z$ to construct the open fattenings $U_{i}$ of $Z_{i} \times\{1\}$ with $\bigcap_{i<\omega} U_{i}=\varnothing$. For each $i<\omega$ there are Euclidean openings $V^{i}(n) \supseteq Z_{i}$ such that $\bigcap_{n<\omega} V^{i}(n) \cap Z=Z_{i}$. Without loss of generality we may assume that, for each $n$ and each $i<j, V^{j}(n) \subseteq V^{i}(n)$. Then, for each $i<\omega, V^{i}(n) \times\{0\} \cup Z_{i} \times\{1\}$ is open in $X$.

Let $U_{i}=V^{i}(i) \times\{0\} \cup Z_{i} \times\{1\}$. It is straightforward to verify that $\bigcap_{i<\omega} U_{i}=$ $\varnothing$.

Claim 4.4. $Z \times\{1\}$ is not $a G_{\delta}$ in $X$.

Proof. It clearly suffices to prove that if $U$ is an open neighborhood of $Z \times\{1\}$ then $Z \times\{0\} \backslash U$ is countable.

Suppose $Y=\{z:(z, 0) \notin U\}$ is uncountable. Fix $D \subseteq Y$ countable dense in the Euclidean topology on $Y$. Then by Lemma 4.2 there is a $y \in Y$ such that $H(y) \cap D$ is infinite. Therefore $(y, 1) \in \overline{D \times\{0\}}$, which is a contradiction.

Unfortunately $X$ is not normal. This follows from the next claim, that $X$ is not countably paracompact, and from the fact that normal, countably metacompact spaces are countably paracompact.

Claim 4.5. $X$ is not countably paracompact.

Proof. Let $\left\{X_{n}: n<\omega\right\}$ be a decreasing sequence of subsets of $Z$ such that in the Euclidean topology, each $X_{n}$ is $\aleph_{1}$-dense in $Z$. Let $A$ be countable and dense in $Z$. Fix $n<\omega$ and $U_{n} \supseteq X_{n} \times\{1\}$ an open subset of $X$. By Lemma 4.2, $\left\{x \in X_{n}: H(x) \cap A\right.$ is finite $\}$ is countable. Therefore, $X_{n}^{\prime}=\{x \in$ $X_{n}: H(x) \cap A$ is infinite $\}$ is $\aleph_{1}$-dense in $Z$. Letting $A^{\prime}=A \times\{0\} \cap U_{n}$, Lemma 4.2 implies that $(X \times\{0\}) \cap \overline{U_{n}}$ is cocountable. Therefore, for any sequence of open sets $U_{n} \supseteq X_{n} \times\{1\}, \bigcap_{n<\omega} \overline{U_{n}} \neq \varnothing$.

In [FM] Fleissner and Miller prove the consistency of the existence of a Q-set concentrated on a countable set. As $\mathfrak{b}=\omega_{1}$ is equivalent to the existence of an uncountable set of reals concentrated on a countable set $[R], \mathfrak{b}=\omega_{1}$ and the existence of a Q-set are mutually consistent with ZFC.

\section{REFERENCES}

[BBu] Z. T. Balogh and D. K. Burke, A total ladder system space by ccc forcing, Topology Appl. 44 (1992), 37-44.

[Bu1] D. K. Burke, Closed discrete subsets in 1 st countable, countably metacompact spaces, Topology Appl. 44 (1992), 63-67.

[Bu2] _ PMEA and first countable, countably metacompact spaces, Proc. Amer. Math. Soc. 92 (1984), 455-460.

[vD] E. K. van Douwen, The integers and topology, Handbook of Set-Theoretic Topology (K. Kunen and J. Vaughan, eds.), North-Holland, Amsterdam, 1984, pp. 111-168.

[FM] W. G. Fleissner and A. Miller, On $Q$ sets, Proc. Amer. Math. Soc. 78 (1980), 280-284.

[H] S. H. Hechler, On the existence of certain cofinal subsets of ${ }^{\omega} \omega$, Proc. Sympos. Pure Math., vol. 13, Amer. Math. Soc., Providence, RI, 1974, pp. 155-173.

[R] F. Rothberger, Sur les familles indenombrables de suites de nombres naturels et les probleme: concernant la propriété C, Proc. Cambridge Philos. Soc. 37 (1941), 109-126. 
[S] P. Simon, A compact Fréchet space whose square is not Fréchet, Comment. Math. Univ. Carolin. 21 (1980), 749-753.

[Sh] S. Shelah, A consistent counterexample in the theory of collectionwise Hausdorff spaces, Israel J. Math. 65 (1989), 219-224.

[T] S. Todorcevic, Partition problems in topology, Contemp. Math., vol. 84, Amer. Math. Soc., Providence, RI, 1989.

Department of Mathematics, University of Toronto, Toronto, Ontario, Canada M5S 1A1

Current address: Department of Mathematics, Ohio University, Athens, Ohio 45701

E-mail address: szeptyckCoucsace.cs.ohiou.edu 